\title{
BEAGLE-A DARWINIAN APPROACH TO PATTERN RECOGNITION
}

\author{
RICHARD FORSYTH \\ Department of Mathematics. Polytechnic of North London, \\ Holloway Road, London N7 8DB (U.K.)
}

(Received December 17, 1980)

\begin{abstract}
"Tlere is grandeur in this view of life, with its several powers, having been originally breathed by the Creator into a few forms or into one; and that whilst this planet has gone cycling on acirrding to the fixed law of gravity, from so simple a beginning endless forms most beautiful and most wonderful have been, and are being evolved."
\end{abstract}

Charles Darwin-The Origin of Species.

\begin{abstract}
BE ;LE (Biological Evolutionary Algorithm Generating Logical Expressions) is a computer package for producing deci, n-rules by induction from a database. It works on the principle of "Naturalistic Selection" whereby rules that fit the nia badly are "killed off" and replaced by "mutations" of better rules or by new rules created by "mating" two bet: adapted rules. The rules are Boolean expressions represented by tree structures.

The software consists of two Pascal programs, HERB (Heuristic Evolutionary Rule Breeder) and LEAF (Logical Exiciator $A$ nd Forecaster). HERB improves a given starting set of rules by running over several simulated generatw... LEAF uses the rules to classify samples from a database where the correct membership may not be known. Pri.mnary tests on three different databases have been carried out-on hospital admissions (classing heart patients as deatis or survivors), on athletic physique (classing Olympic finalists as long-distance runners or sprinters) and on foot $:$ i: results (categorizing games into draws and non-draws)

It :ppears from the tests that the method works better than the standard discriminant analysis technique based on a line.: discriminant function, and hence that this long-neglected approach warrants further investigation.
\end{abstract}

\section{INTRODUCTION}

This report describes $B E A G L E$ (Biological Evolutionary Algorithm Generating Logical Expressions) which is a computer system for producing decision-rules by induction from a database. It works on the principle of natural -or at least naturalistic--selection. Thus it represents a weaving-together of strands in the thought of three great 19th-century Englishmen, Boole, Babbage and Darwin.

While "knowledge engineering" or "knowledge refining" is currently enjoying something of a vogue and has already begun to produce impressive results. ${ }^{1}$ this report contains a plea not to neglect a parallel endeavour with a less mechan-

หหร. 103 . istic flavour that we might call "knowledge farming" or perhaps "sophiculture". In particular it is the author's contention that the great principle of natural selection is a valuable tool in the stock-intrade of the conscientious knowledge engineer (or farmer).

The idea of systems that improve by a computational analogy with survival of the fittest has been pursued before, ${ }^{2-4}$ but has lapsed from favour somewhat since the pioneering spirit of Cybernetics was consolidated into the mature (?) discipline of Artificial Intelligence. Selfridge's "Pandemonium" was an early example 5 of a system designed to contain "the seeds of selfimprovement" which involved, among other things, replacing "demons" which discriminated 
poorly among the input patterns they were supposed to distinguish with new "demons" formed by randomly altering the parameters of surviving ones. But probably the only really thoroughgoing attempt to "breed" intelligence in the abstract was by Barricelli and Bell. ${ }^{6}$

Barricelli's "symbioorganisms" were sequences of integers that existed in a universe consisting of an array of cells. Whenever two organisms both attempted to expand into the same space a game of Tac-Tix was played between them to the death. The number patterns of the organisms were interpreted as moves in the game. The surviving organisms were allowed to reproduce (asexually,
The datafile contains a "training set" of samples for which the categories are known. It should begin with two integers, $W$ and $F$. W is the width in characters of the description field for each sample (0 if absent). $F$ is the number of features. Then follows the data-for each case the description field of $\mathrm{W}$ characters, $\mathrm{F}$ numbers which are measures for the case on each feature or variable (integers only at present with at least one space or new line to separate them), and lastly a number indicating the actual category to which that case belongs. (The category number must end a line.)

There follows the first three lines of a typical datafile.

\section{8 \\ $5176816512114889573 \quad 17 \quad 14166 \quad 115225110562 \quad 206 \quad 113 \quad 340$ 1}

it appears) and some random mutations introduced, after which the process was repeated. After some thousands of generations he had a collection of organisms that were expert at Tac-tix. Barricelli found it quite an effective technique, and it is my view that it is due for a revival.

\section{BEAGLE-THE USER'S VIEW}

The system as presently implemented consists of two PASCAL programs running on the DEC System-10 at Polytechnic of North London, namely $H E R B$ (Heuristic Evolutionary Rule Breeder) and LEAF (Logical Evaluator And Forecaster). They can be accessed like any other statistical package and in function correspond most closely to discriminant analysis. Together they perform the task of classifying samples into one of two or more categories on the basis of the values of a number of measures or parameters describing each sample. $H E R B$ creates and/or modifies the classification rules which $L E A F$ then uses, typically to forecast group membership for samples whose class is not known.

\subsection{The HERB Program}

$H E R B$ requires three input files - a datafile, a payoff file and an old rule file (possibly empty). It produces as output a new rule file which is as good as or better than the old one.
This is the beginning of a file of data from 113 patients admitted to hospital with heart complaints. ${ }^{7}$ Each patient was measured on 18 variables on admission. Preceding the 18 scores is an identification number (4 characters) which is 517 for this patient. Following the scores is the category number $(1=$ lived, 2 = died). These cases were used for testing: see section 4 . (The first 3 variables are age, height and sex; so this patient was 68 years old, $165 \mathrm{~cm}$ tall, and male... he survived.)

To enable the program to assess each rule's performance the user must also furnish a payoff matrix in a separate file which effectively states the value or cost of each classification or misclassification. The payoff file also indicates how many categories are in use. Since the program works on tri-state logic where $1=$ yes, $0=$ don't-know and $-1=$ no, this means a 3 by $\mathrm{NC}$ table where $\mathrm{NC}$ is the number of classes. (Later releases will allow the user to specify one of several multi-state bogics of which 0.1 . Boolean. will be a special case.)

For the tests on the hospital admission data the payoff matrix was as follows.

$\begin{array}{ccc} & \text { Actual Class } \\ \text { Computer Decision } & 1 \text { (lived) } & 2 \text { (died) } \\ -1 \text { (no) } & -1 & +1 \\ 0 \text { (maybe) } & 0 & 0 \\ 1 \text { (yes) } & +1 & -1\end{array}$


Thus a rule gained a point for a correct classification and lost one for an incorrect one. More complex reward/punishment schedules with more classes are of course possible.

Finaliy, the user supplies an initial rule file containing up to 64 rules. Initially there may be none, in which case the program will generate some at random

A ruic is represented by a fully bracketed Boolean expression ended by a dollar sign, such as

$$
\text { ((\#4 GE 20) OL ((\#4 LT 10) AN (\#17 NE 0)))\$ }
$$

which sit:es that variable 4 (\#4) should exceed or equal 2 or that both variable 4 should be less than 10 and variable 17 not zero for the rule to give a pi sitive (true) result.

The werators are as follows.

$\begin{array}{ll}\text { EQ } & \text { arithmetic equality } \\ \text { NE } & \text { arithmetic inequality } \\ \text { GT } & \text { greater than } \\ \text { LT } & \text { less than } \\ \text { GE } & \text { greater than or equal to } \\ \text { LE } & \text { less than or equal to } \\ \text { OL } & \text { logical disjunction (inclusive or) } \\ \text { AN } & \text { logical conjunction (and) } \\ \text { NO } & \text { negation } \\ \text { PLES } & \text { addition } \\ \text { LESS } & \text { subtraction } \\ \text { BY } & \text { multiplication } \\ \text { OVER } & \text { division }\end{array}$

(The odi names such as $\mathrm{AN}$ and $\mathrm{OL}$ were chosen to avois? \& clash with $P A S C A L$ predefined operators.) same format as the training set-the only difference being that the actual classes need not be known zero indicating unknown class membership-and runs a rule file on it. The user specifies how many rules to use: these are always left ordered by $H E R B$ with the best first. $L E A F$ can be requested to produce: (1) a listing of all cases with predicted class, and actual class and score if known; (2) a summary of the performance of each rule and all the rules jointly: (3) an ordering of cases by rule consensus from most likely Yes to most likely No.

Notice that the rules produced by $H E R B$ can be applied by a person. $L E A F$ is merely a convenience. Contrast this with the linear functions with coefficients expressed to 8 or 10 decimal places output by conventional discriminant analysis packages: no one in their right mind would try to use those without machine assistance.

\section{HOW HERB WORKS}

HERB attempts to mimic evolution by natural selection. Its "organisms" are the rules and their survival depends on how well they categorize the samples in the training set.

It runs for a number of generations. chosen by the user. A gencration consists of one run through the data during which each rule is evaluated on every case and scored according to the payoff matrix. The rules are then ranked by total score with the best rules at the top, i.e. those with the highest score.

The scoring formula is actually

$(($ GOODNESS-MINSCORE) $* 100 *$ GFACTOR $)$ (MAXSCORE-MINSCORE)-SIZE

Arithmetic is integrated with logical evaluation because the three truth values are $+1,0$ and -1 . If a rule yields a final value outisde the logic range it will be truncated to the nearest extreme. Arithmetic subexpressions are not truncated (unless they would cause overflow).

\subsection{The LEAF Program}

LEAF is far simpler. It takes a datafile in the where MINSCORE and MAXSCORE are the lowest and highest scores possible, GOODNESS is the accumulated payoff and SIZE is the size of the rule measured by counting nodes (terms or subexpressions). What this means is that a longwinded rule scoring the same as a more concise one will be ranked lower. Remember we are treating the rules as organisms: the larger animals need more "food". GFACTOR can be set by the user to alter the balance between goodness and size. A high GFACTOR asks for a good rule at, 
almost, any price; a low setting is a bias towards brevity.

Having been ranked thus, the breeding begins. The top quarter $(25 \%)$ are left alone. They are good enough to survive untouched. The second quarter are all subjected to a procedure GROW which adds a node composed at random. For example, GROW on

$$
\text { ((\#1 OL (\#2 EQ 0)) GT 62) }
$$

might produce

$$
\text { ((\#1 PLUS 5) OL (\#2 EQ 0)) GT 62)). }
$$

Rules in the third quarter are subjected to a procedure named SLIM which is the obverse of GROW: they lose a randomly selected term or subexpression. They have survived but are suffering from "malnutrition". Finally the bottom $25 \%$ are subjected to a procedure called KILL which, squeamish readers may be assured, causes no pain.

To replace the dead rules new ones are formed by mating together elements from the top half of the list. Internally the rules are held as binary trees. The MATE procedure takes a random subtree from one parent rule selected at random from the upper half and combines it with another chosen likewise. The two parts are then linked by a randomly selected connective to give a fully formed expression. For example, the mating of

$$
((\# 4 \text { GT 62) AN (\#3 EQ 0)) }
$$

with so on, leaving the pruned tree with the same value but expressed more succinctly. The result of TIDYing

$$
\text { (((5 BY 4) GT 16) AN (\#17 EQ \#8)) }
$$

would be

$$
\text { (\#17 EQ \#8) }
$$

since $(5 \mathrm{BY} 4)=20$ and $(20 \mathrm{GT} \mathrm{16})=+1$ (true).

Then the next generation begins. The process continues for the required number of generations, and then the new rules are printed onto the output file.

\section{SOME TESTS OF HERB}

The question is: does it work?

To establish a comparative standard the discriminant analysis function of the SPSS package on the DEC System-10 library was run with the hospital admission data. It produced two linear functions of seven variables plus a constant. Both these functions are to be evaluated for each case and if function 1 gives a higher value the sample is assigned to group 1 (living) whereas if function 2 gives a higher value the sample is assigned to group 2 (dead). (There were 70 survivors and 43 deaths, but this information was not used to weight the prior probabilities.)

The diagnostic variables chosen were, in descending order of importance, numbers 6 (mean arterial pressure), 9 (mean venous pressure), 4

\section{((\#17 BY -2) PLUS ((\#15 GT 5) OL (\#2 LE \#8)))}

might result in

$$
\text { ((\#4 GT 62) LESS \#8). }
$$

The next step is to apply the MUTATION procedure to a few (randomly selected) of the lower $7 / 8$ ths of the rule list. This can do various things like altering terms, swapping subtrees, altering operators and so forth. (The top $1 / 8$ th is inviolate: rules that high can only be changed if a better 'strain' displaces them.)

Finally, procedure TIDY is applied to all rules. This cuts down redundancies such as double negatives, expressions with a constant value and (shock type), 14 (urinary output), 10 (body surface area), 15 (plasma volume index) and 16 (red cel'. index). All were positively loaded on function 1 except 9 (venous pressure). The CPU time to generate these results was 2.85 seconds.

When re-run on the training set data the discriminant functions correctly classified $75 \%$ of the cases. The mistakes were: 16 of group 1 classed as group 2; 12 of group 2 classed in group 1 .

The $H E R B$ program was then run on the same data, starting completely from scratch-i.e. with no pre-determined rules. For all the tests the number of ruler was fixed at 48. After 111 gener- 
ations a run of $L E A F$ indicated that the top rule was correctly grouping $73 \%$ of the cases in the training set. This took about 2 minutes of runtime.

After 5010 generations the top rule was correct on $81 \%$ the cases (counting a 0 , or don't-know, as incorrect as well as any outright misclassifications).

The tor rule at this stage was

$$
\text { (\#6 GE (61 LESS \#14)) }
$$

where $\Rightarrow t$ is mean arterial pressure and \#14 is urinary o.:put. What it says is that if mean arterial press:.: $(\mathrm{mm} \mathrm{Hg})$ is greater than or equal to urinary itput $(\mathrm{ml} / \mathrm{hr}$ ) subtracted from 61 the patient sould survive, otherwise the patient is likely to 3.2 . Its mistakes were: 2 survivors classed as grouf :: 20 deaths classed as group 1. (The payoff ma:rix could have been adjusted if these different a.nds of error were not equally costly, as no doubt would be the case in practice.)

It is noidble that already we have a rule that is better thi:: the linear discriminant functions: and so much simpler that a hospital orderly could easily apry it. (Is this a danger?)

Perhap statisticians, who are on the whole quite cor:ant to computerize techniques worked out by P: :son and Fisher over 50 years ago and who tend (i) regard even Bayesian decision-making as ia: exciting but not every respectable novelty. sould wake up to the potential of today's evert systems.

A second test was run on data concerned with the physicue of male athletes. Here the data was the age $:=1$ ), height in inches (\#2), weight in pounds $(=3)$ and race $(\# 4)$ of the medallists in the runing and walking events of the 1968 Mexico Olympic Games. Race was either 0 (white) or 1 (black). (One Japanese was arbitrarily assigned io race 0 and Mohammed Gammoudi, who appeired twice by virtue of winning medals in two difirent events, was classed as 0 the first time and i the next: he is Tunisian.)

The aim was to arrive at a rule that would distinguisis the sprinters from the long-distance men on the basis of the data about age, height, weight and race. The events were actually put into 5 classes, from shortest to longest.

$\begin{array}{cl}\text { Class } & \text { Events } \\ 1 & 100 \mathrm{~m}, 200 \mathrm{~m}, 110 \mathrm{~m} \text { hurdles } \\ 2 & 400 \mathrm{~m}, 400 \mathrm{~m} \text { hurdles } \\ 3 & 800 \mathrm{~m}, 1500 \mathrm{~m}, 3000 \mathrm{~m} \text { steeplechase } \\ 4 & 5000 \mathrm{~m}, 10000 \mathrm{~m}, 20 \mathrm{~km} \text { walk } \\ 5 & \text { Marathon, } 50 \mathrm{~km} \text { walk }\end{array}$

The various payoffs were assigned accordingly.

\begin{tabular}{rrrrrr} 
& \multicolumn{7}{c}{ Actual Class } \\
Rule Decision & 1 & 2 & 3 & 4 & 5 \\
-1 & 2 & 1 & 0 & -1 & -2 \\
0 & 0 & 0 & 0 & 0 & 0 \\
+1 & -2 & -1 & 0 & 1 & 2
\end{tabular}

A decision of +1 is interpreted as long-distance competitor, -1 as sprinter.

After 666 generations the top rule was

$$
\text { ((155 LESS \#3) PLUS (-5BY \#4)) }
$$

which was only making one mistake on the 32 samples in the training set. What it says, in brief, is that if you are white and weigh over 155 pounds you are a sprinter, if you weigh less you are a distance runner: if you are black and weigh over 150 pounds you are a sprinter. otherwise you are a long-distance runner.

As a test 12 gold medallists from the 1980 Moscow Olympics were rated by this rule. This was fresh data, not used in the training phase. All were correctly categorized except Pietro Mennea who, at 150 pounds, is a bit light for a white sprinter.

N.B. These figures apply to Olympic athletes: just because you weigh over 155 pounds do not get the idea that you are a match for Allan Wells!

\section{REMARKS}

I see three justifications for this kind of exercise. Firstly, it is interesting in its own right; secondly, the rules behave in an interesting fashion; and thirdly, it seems to work.

In the first place it is fun to try a little abstract gardening, growing an orchard of binary trees. And it might be fruitful in another sense. After all, we are only here by courtesy of the principle of natural selection, AI workers included, and since it is so powerful in producing natural intelligence 
it behoves us to consider it as a method for cultivating the artificial variety.

The second justification is the surprisingly lifelike behaviour of the rules themselves. It can be appreciated by looking at the top 4 rules produced by $H E R B$ on the hospital admissions data after $1,11,111$ and 1111 generations. over a large mass of figures and extract an efficient classification rule for the cases there would be little need for this kind of program.)

The third point is that the system works quite well, even though this is version 1.0 of the program. The rules produced are short and to the point, though it is fair to mention that the com-

$$
\begin{aligned}
& 1 \text { generation } \\
& (\# 1 \text { PLUS 0) } \\
& (2 \text { PLUS 0) } \\
& (\# 1 \text { PLUS 0) } \\
& (\# 1 \text { PLUS 0) } \\
& \text { [27 = chance expectation] }
\end{aligned}
$$

11 generations

$$
\begin{aligned}
& (\mathrm{NO}((\# 16 \mathrm{LE}-1) \mathrm{BY}(\# 6 \mathrm{GT} 53))) \\
& (\# 6 \mathrm{GE} \# 1) \\
& (((\# 16 \mathrm{LE}-1) \mathrm{BY}(\# 6 \mathrm{GT} 53)) \mathrm{LT} \\
& ((\# 1 \mathrm{EQ} \# 11) \mathrm{NE}-10000))
\end{aligned}
$$$$
\text { (\#1 PLUS 0) }
$$

111 generations

(137 OVER (\#6 GT 53))

(135 OVER (\#6 GT 53))

(1 OVER (\#6 GT 53))

(137 OVER (\#6 GT 53))

1111 generations

(\#6 GE (61 LESS \#14))

(\#6 GE (62 LESS \# 14))

((61 LESS \#14) LE \#6)

((61 LESS \#14) LT \#6)

What we see here is the appearance (and subsequent disappearance) of dominant "species". Each type flourishes for some time until quite suddenly supplanted by a new and superior line-typically a mutation of one of its own offspring When this happens the extinction of the more primitive forms is rapid and complete.

It seems that once a rule fastens on a particular indicator variable or combination of variables it will give rise to several copies or near-copies forming a family which thrive until a better rule appears, possibly using an entirely different set of indicators. It is as if the new variety have found a more nourishing "diet".

There is nothing to prevent the user inserting a man-made rule at any stage; indeed it is salutory to do so since all trace of it is normally lost within a few generations. (If it were easy to cast your eye

$\begin{array}{cc}\text { Age } & \text { Score } \\ 1 & 27 \\ 1 & 27 \\ 1 & 27 \\ 1 & 27 \\ & \\ & \\ 5 & 49 \\ 3 & 40 \\ & \\ 4 & 39 \\ 10 & 27 \\ & \\ 77 & 51 \\ 55 & 51 \\ 41 & 51 \\ 33 & 51 \\ & \\ 691 & 69 \\ 502 & 69 \\ 479 & 69 \\ 478 & 69\end{array}$

puting cost is quite heavy--almost 2 minutes of runtime per 100 generations on the DEC System-10 (KL 10 processor) on the hospital data.

As BEAGLE is quite successful on toy databases the reader with gambling instincts may care to participate in a little field testing on far more messy data. For what it is worth, here is the top rule produced by $H E R B$ after 400 generations on a datafile containing 1000 English and Scottish League and Cup football results (1979--80).

$$
\text { (NO ((\#58 OVER \#45) AN \#77)) }
$$

Its value is meant to be true (positive) for drawn games, negative otherwise. The variables are: \#58 the away team's ground capacity (in thousands of spectators) subtracted from the home team's crowd capacity (thousands): \#45 goals scored by the away side in their last away game: 
\#77 diterence formed by adding home team's home away te.m's goals conceded in their last 8 away games and subtracting home team's goals against plus aw: team's goals for in the same matches.

\section{FLTLRE DIRECTIONS}

BEAliLE is still at a prototype stage, and can be consicrably improved. One planned enhancement $u$.: mentioned in section 2.1-allowing the multi-st..te logic range to be specified by the user.

A scond extension that would not be difficult to imp.iment would be to allow floating-point arithm:... as well as integers, though the interaction w.: logical values would have to be carefully conside..ed first. It might be an opportunity ${ }^{8}$ to introdu.: 'fuzzy logic' at the same time. (HERB and $L E \& F$ already use a "slightly fuzzy" comparison schume such that $64 \mathrm{GE} 65$ is not quite so false ar GE 65, but the usefulness of this has not bee:. assessed.)

Ano:...: planned improvement is the inclusion of add:..unal operators. The MOD (remainder) functio:: will be one, but more important will be the pai: $\$ O \ldots$ OS to allow constructions of the form

\section{(B SO (A1 OS A2))}

which s..ves for

\section{if $B$ then $A 1$ else $A 2$}

and wh: sive a rudimentary programming ability Of cou: $:$ this highlights the fact that the rules are really prams in a special-purpose language, which a...sht lead to the conclusion that the system shrild ultimately generate LISP functions. But thi :s rather a distant goal. It would remove all restristions, but whether $H E R B$ or anything like it culd cope with the extra power remains to be seen. Probably a compromise. such as generalizing $i: 2$ rules as far as production systems of a limited complexity, would be more manageable.

In effict the rules as they stand simulate singlecelled $v$ ganisms, without specialization of function. Tu move on to a hierarchic structure, corresponding to multi-cellular animals $H E R B$ would need 2nd-level organisms (strategies) with "green fingers" whose welfare depended on successfully managing the first-level ones.

A more serious need is to make greater use of information provided by bad rules, rther than just discarding them and quite possibly regenerating them later. This is a major weakness and all the obvious remedies (e.g. a rote memory of bad rules) would introduce considerable overheads.

\section{CONCLUSIONS}

I would like to present Naturalistic Selection as a viable AI technique. This is not to say it is a panacea. I suspect that there is alwals a better way (cheaper and/or quicker); but no single method is more appropriate for "satisficing" in such a wide variety of problems. ${ }^{9}$

On the credit side Naturalistic Selection is absolutely general. A user can hurl any data at $H E R B$ however nonlinear, however "noisy", however much it violates the assumptions about distribution and scaling underlying most statistical tests. and get a reasonable set of discrimination rules in reasonable time. And since those rules are public and comprehensible. not arcane technocratic black magic, man-machine cooperation is facilitated. The human can do some of the hypothesizing (which people are good at) leaving the testing (which people are bad at) to the computer.

For example, to classify the test data used in this paper a sequential decision procedure such as that proposed by Hunt ${ }^{10}$ might have been more economical; but the trouble with stepwise algorithms which yield a discrimination net or progressive filter network is their susceptibility to noise in the data. They work best in situations where there can be no error in the training data and where the variables have rather few discrete values (e.g. chess endgames). HERB though rarely if ever optimal. will almost always come up with something usable.

Lastly there is the matter of image. Is the designer of expert systems to be seen as a soulless white-coated machine-minder or as someone who, for the first time since the expulsion from Eden, is not merely picking new fruit from the forbidden (Binary) Tree of Knowledge, but actually making it grow? 
[PASCAL source listings of $H E R B$ and $L E A F$ are available on request from the author at: Maths Dept., Polytechnic of North London, N7 8DB, U.K.]

\section{REFERENCES}

1. B. G. Buchanan and T. M. Mitchell "Model-Directed Learning of Production Rules" in Waterman \& Hayes Roth (eds) Pattern-Directed Inference Systems (Academic Press, New York, 1978.

2. G. Pask, An Approach to Cybernetics (Hutchinson, London, 1961).
3. A. Bernstein and I. Rubin. "Artificial Evolution of Problem-Solvers" American Behatioural Scientist (May, 1965).

4. L. J. Fogel et al., Artificial Intelligence through Simulated Evolution (Wiley, New York, 1966).

5. O. G. Selfridge, "Pandemonium a Paradigm for Learning" N.P.L Symposium on Mechanization of Thought Processes (HMSO, London, 1959).

6. A. G. Bell, Games Plaving with Computers (Allen \& Unwin, London. 1972)

7. A. A. Afizi and S. P. Azen, Statistical Analysis: A Computer Oriented Approuch (Academic Press, New York, 1972).

8. L. A. Zadeh, "Fuzzy Sets" Information and Control 8 (1965).

9. H. A. Simon. The Science of the Artificial (MIT Press, Mass., 1966).

10. E. B. Hunt et al. Experiments in Induction (Academic Press. New York. 1966). 